Pensamiento Crítico N. ${ }^{\circ} 9$, pp. 45-59

\title{
Efectos de la presencia de la banca ex- tranjera en la banca nacional del Perú: un estudio comparativo 1995-2007 (avances)
}

Mg. Gaby Cortez Cortez

\section{RESUMEN}

La presencia de bancos extranjeros en la economía peruana no es un fenómeno nuevo. Sin embargo, su importancia se pone de manifiesto en las últimas décadas con la apertura de la economía y la liberalización del mercado bancario.

El propósito del presente trabajo es evaluar los efectos del ingreso de bancos extranjeros en el comportamiento de los bancos en el Perú en relación a la competencia, eficiencia, márgenes de interés, y la ganancia.

Se puede plantear la hipótesis de que el ingreso de los bancos extranjeros en el Perú, contribuye a estimular a los bancos nacionales a través de una mayor competencia, menores márgenes, disminución de costos, al aumento de la eficiencia y a la mayor diversidad de productos y servicios financieros.

Palabras clave: Bancos extranjeros, competencia, margen de interés, ganancia. 


\title{
Gaby Cortez Cortez
}

\begin{abstract}
The presence of foreign banks in the Peruvian economy is not a new phenomenon. However, its importance is reflected in the recent decades with the opening of the economy and the liberalization of the banking market. The purpose of this study is to assess the effects of foreign banks entry in the behavior of banks in Peru in relation to competition, efficiency, interest margin, and profit. We hypothesize that the foreign banks entry in Peru helps to stimulate domestic banks through greater competition, lower margins, reduced costs, increased efficiency and greater diversity of products and financial services.
\end{abstract}

Keywords: Foreign banking; competition; interest margin; profit.

\section{INTRODUCCIÓN}

La internacionalización y liberalización del sistema financiero llevada a cabo en las últimas décadas permitió el ingreso de bancos extranjeros en casi todas las economías del mundo. América Latina y el Perú no estuvieron ajenos a este fenómeno que se caracterizó por la adquisición de bancos importantes y líderes del sistema. El Banco Continental es un claro ejemplo de esta estrategia de compra de un banco líder del sistema por un banco extranjero, el español Banco Bilbao Vizcaya.

También, el ingreso del importante banco español Santander fue un intento de penetración del mercado bancario peruano con resultados no favorables. Igualmente, la banca chilena ha intentado tener una presencia en el mercado peruano con resultados en algunos casos nada favorables como el de los Bancos del País, Serbanco, Bancosur, Solventa y República, dirigidos básicamente al crédito de consumo. El único banco que sigue operando es el Banco del Trabajo, pero con claros indicios de ser tomado por el reciente ingresado banco extranjero Scotiabank de origen canadiense.

Otros bancos pequeños de origen extranjero que se mantienen en el sistema son el Banco Financiero, de capitales ecuatorianos, y el Banco Interamericano de Finanzas, perteneciente al grupo Fierro de España.

Esta dinámica de adquisición y penetración de bancos extranjeros se sigue manteniendo actualmente. El canadiense Scotiabank ingresó tímidamente al mercado perua- 


\section{efectos de la presencia de la banca extranjera en la banca nacional del Perú}

no con la compra de una parte de las acciones del Banco Sudamericano (30\%). Posteriormente, este banco ante los problemas de mala gestión surgidos en el Banco Wiese Sudameris aprovechó la oportunidad para hacerse de un banco igualmente importante del sistema; ingresando al mercado con una nueva estrategia de manejo de su propia marca. Recientemente, han hecho su ingreso dos nuevos bancos el Hong Kong Shangai Bank Corporation (HSBC) de origen inglés y el chileno Falabella.

De esta forma, se puede concluir que la presencia de la banca extranjera en el Perú no solo es importante por su número, sino que además tiene un poder de mercado muy importante, planteando la interrogante sobre su desempeño, desarrollo y estabilidad del sistema financiero peruano.

\section{REVISIÓN DE LA LITERATURA}

Existe importante literatura sobre la presencia de la banca extranjera en las economías tanto de países desarrollados como en desarrollo. Los estudios ocupan un amplio espectro desde la estabilidad del sistema financiero, hasta el análisis del margen, y los costos de los bancos.

Eduado Levy Yeyati y Alejandro Micco (Yeyati, 2007) analizan las implicancias que el proceso de concentración y la presencia extranjera provocan en la conducta competitiva de los bancos, y en la estabilidad del sistema financiero en diversos países de Latinoamérica. El estudio muestra que el incremento de la concentración no debilita la competencia en la región y que la presencia extranjera parece conducir a una menor competencia en el mercado bancario, aunque para estos autores, no esta claro el argumento analítico que soporte este punto de vista en la literatura sobre el tema.

Autores como Eric Van Tassel y Sharmila Vishwasrao (Tassel, 2007) están interesados en los incentivos que tienen los bancos para operar en otros mercados liberalizados. $\mathrm{El}$ argumento que exponen es que los bancos extranjeros con costos más bajos están probablemente menos informados que los bancos domésticos, sobre la situación del mercado local de crédito y sus riesgos inherentes. Esta ventaja relativa puede generar incentivos para que los bancos extranjeros negocien la adquisición de los bancos domésticos, con el propósito de capturar la información que estos bancos disponen. 


\section{Gaby Cortez Cortez}

Sin embargo, es difícil evaluar el valor de la información que disponen los bancos domésticos, de tal manera que los bancos extranjeros calcularán el modo óptimo de entrar al país y que precio pagar por sus adquisiciones. Esta elección tiene implicancias para la sobrevivencia de los bancos domésticos, y la forma en que los bancos extranjeros se ubican dentro de un mercado liberalizado.

Otros autores como Fotios Pasiouras y K. Kosmidou (Pasiouras, 2007) están interesados en ver cómo las características especificas de los bancos y el entorno afectan los ingresos de los bancos domésticos y extranjeros. Los resultados indican que los beneficios de los bancos, tanto domésticos como extranjeros, están afectados no sólo por las características específicas de los bancos, sino también por la estructura del mercado financiero y las condiciones macroeconómicas.

El estudio efectuado por Hidenobu Okuda y S. Rungsomboon (Okuda, 2006) sobre la presencia de la banca extranjera en Tailandia, investiga el cambio en la tecnología de producción de los bancos extranjeros y domésticos sugiriendo que los bancos extranjeros y domésticos tiene diferencias en sus ventajas de información y procesamiento. Después de la crisis de Asia, la aplicación de medidas más estrictas en la regulación mejora el rendimiento de los costos comparativos de los bancos extranjeros. La adquisición de los bancos domésticos por bancos extranjeros reducen los costos asociados con la entrada de los bancos y el mejoramiento de su eficiencia operacional.

La investigación de los efectos en el corto plazo de la entrada de bancos extranjeros en la conducta del sector bancario doméstico condujo a Robert Lensink y N. Hermes (Lensink, 2004) a formular la hipótesis de que estos efectos dependen del nivel de desarrollo económico del país anfitrión. La investigación muestra que un nivel bajo de desarrollo económico del país donde ingresan los bancos extranjeros está generalmente asociado con altos costos operativos y altos márgenes de los bancos domésticos. La entrada de bancos extranjeros está asociada con una caída de los costos, los beneficios y márgenes de los bancos domésticos.

En un estudio sobre Filipinas, Angelo A. Unite y Michael J. Sullivan (Unite, 2003) encuentran evidencia que la entrada de bancos extranjeros está asociada con una reducción en los márgenes de interés y los beneficios bancarios. El ingreso de bancos extranjeros ha llevado a un mejoramiento de la competencia obligando a los bancos domésticos a ser más eficientes. 


\section{efectos de la presencia de la banca extranjera en la banca nacional del Perú}

\section{BREVE RESEÑA HISTÓRICA DE LA PRESENCIA DE LA BANCA EX- TRANJERA EN LA ECONOMÍA PERUANA}

Hasta los dos primeros años de los noventa, el sector bancario peruano estaba compuesto por bancos comerciales privados, y por bancos estatales (asociados, regionales, de fomento y el Banco de la Nación). El Banco Central de Reserva dirigía la mayor parte de sus colocaciones a proporcionar recursos al Banco de la Nación y a la Banca de Fomento, especialmente. A través del proceso de privatización, se vendieron dos bancos asociados grandes (Continental e Interbank), y se liquidó el Banco Popular.

En abril de 1995, el Estado peruano vende el Banco Continental al consorcio formado por el banco español Banco Bilbao-Vizcaya y el Grupo Brescia de capitales peruanos por 256 millones de dólares. Desde un comienzo, se percibió la intención de este banco español de ingresar al mercado peruano con la estrategia de tomar un banco líder del sistema, para facilitar su penetración en el mercado local, así como contar con la cartera de clientes, la experiencia en el manejo de la plaza local y una red de sucursales y agencias en todo el Perú. Esta situación ventajosa se vio reforzada años antes cuando el Estado peruano transfirió al Banco Continental los bancos regionales Banco Nor-Perú, Banco de los Andes y el Banco Amazónico. La estrategia del Continental (BBV) lo llevaba a obtener una ventaja con la que accedería a una penetración y crecimiento más rápido que si empezaba desde abajo como un nuevo banco en el mercado local peruano, ya que esta adquisición le permitía contar con una marca reconocida en el mercado.

En ese mismo año, el banco español Santander ingresa al mercado peruano mediante la compra de dos bancos pequeños: el Banco Interandino y el Banco Mercantil. La estrategia en este caso es el ingreso con una marca propia, y, por lo tanto, le debe tomar más tiempo y esfuerzo consolidarse en el mercado local. Siguiendo esta estrategia, el Banco Santander, en el año de 1999, adquiere el Banco Sur, que previamente, en el año 1996, se había fusionado con el Banco del Libertador. A la luz de los resultados obtenidos, se comprueba que esta estrategia no le permitió al Banco Santander consolidarse en el mercado bancario local, pues años después tuvo que salir del mercado.

Durante 1994, se autorizó el funcionamiento de dos nuevos bancos orientados, principalmente, al otorgamiento de créditos para el consumo: el Banco del Trabajo y 


\section{Gaby Cortez Cortez}

el Banco Solventa. El Banco del Trabajo, cuyo principal accionista es Inversiones Altas Cumbres S.A., que está vinculado al grupo chileno Cummins. El Banco Solventa tuvo una incursión muy breve en el mercado peruano de solo cuatro años.

En los dos años siguientes, empezaron a operar el Banco Serbanco, también con accionistas mayoritarios chilenos, pertenecientes al Grupo Altas Cumbres; y el Bank of Boston, con capitales norteamericanos pertenecientes al First National Bank of Boston.

En el periodo 1993-1995, el PBI real creció a una tasa promedio anual de 8.7\% explicado por el aumento de la demanda global, que se expandió a una tasa promedio anual de $10.1 \%$. Es importante señalar que durante estos años se generó un auge del crédito que se incrementó a una tasa anual de $34.1 \%$, que fue facilitado por la disponibilidad de recursos en moneda extranjera. Pero también se empieza a vislumbrar los inicios de una desaceleración de la economía y la amenaza de una crisis futura debido a la expansión del crédito del sistema bancario al sector privado. Sin embargo, las expectativas de crecimiento de la economía llevaron a los agentes económicos a continuar con la inercia de los años anteriores, especialmente en lo que se refirió a las decisiones de consumo.

En el periodo siguiente (1996-1997), se aprecia un deterioro de la actividad económica con un crecimiento del PBI de apenas $0.4 \%$; la inversión privada cae considerablemente a una tasa promedio anual de $9.1 \%$, determinada por las expectativas de menor crecimiento económico y por problemas de sobreendeudamiento de las empresas, así como por el efecto de la crisis asiática, que condujo a una menor demanda de materias primas del país como los productos minerales y derivados del pescado. $\mathrm{El}$ mercado bancario no podía estar ajeno a estas circunstancias, y se empieza a generar un proceso de fusiones y adquisiciones que llegaron a afectar a varios bancos importantes del sistema.

En 1998, en plena crisis del sistema financiero, es comprado un banco de considerable participación en el mercado bancario peruano como era el Banco de Lima, el cual fue adquirido por el Banco Sudameris, filial del Grupo Banca Comerciale di Italia. El banco Extebandes es adquirido por el Banco Standard Chartered, perteneciente al Grupo Standard Chartered de Gran Bretaña; y el Banco República es intervenido, ingresando a un proceso de liquidación. 


\section{Efectos de la presencia de la banca extranjera en la banca nacional del Perú}

$\mathrm{Al}$ año siguiente, en medio de la recesión económica e ineficiencias en su gestión gerencial, el segundo banco nacional más importante del sistema, el Banco Wiese, fue adquirido por el Banco de Lima Sudameris, creándose el Banco Wiese - Sudameris, manejado por el Holding Lima - Sudameris, perteneciente al grupo italiano Intesa-Bci. El banco Norbank adquiere el Banco del Progreso y también se crea un nuevo banco el BNP - Andes, perteneciente a la Banque Nationale de Paris (Francia).

En el año 2000, se empieza a notar ya una depuración y reordenamiento del mercado bancario con la salida del Banco Latino, el NBK Bank, el Banco Serbanco y el Banco Orión. El Banco Santander se transforma en el Banco Santander Central Hispano luego de su fusión con el Banco Central Hispano de España.

A partir del 2001, el mercado bancario queda reducido a 15 bancos; para luego continuar concentrándose, hasta llegar a 11 bancos en el 2006; y a 13 bancos, en el 2007. Los bancos que salieron del sistema fueron los más pequeños, los que pudieron participar del mercado en la medida que los bancos más grandes no estaban interesados en los nichos que estos activaban, pero una vez que los bancos grandes ingresan a la competencia, estos no tienen otra alternativa que abandonar el mercado. Aquí, cabe mencionar la intención de los bancos pequeños conformados por capitales chilenos, de mantenerse en el mercado, pero que tuvieron poco éxito, excepto en el caso del Banco del Trabajo, que sigue operando en el mercado, pero con una clara intención de ser adquirido por un nuevo participante, el canadiense Scotiabank.

Este banco, Scotiabank, ingresa tímidamente en el mercado peruano adquiriendo acciones del Banco Sudamericano (Bank of Nova Scotia 22.3\% y Scotia South America Limited 7.7\%). Sin embargo, aquí no quedaron sus intenciones y aprovechó para cambiar sus estrategias, y comprar un banco grande del sistema, el Wiese Sudameris, para participar con su propia marca, Scotiabank.

Con la intención de aprovechar los espacios vacíos dejados por los bancos que salieron del sistema, nuevos bancos extranjeros están tratando de colocarse en el mercado peruano, tal es así que inicia sus operaciones para crédito al consumo el Banco Falabella, en un nuevo intento de posicionamiento de capitales chilenos en el mercado bancario peruano. 


\section{Gaby Cortez Cortez}

Finalmente, en el 2006, abre sus puertas un nuevo banco el Hong Kong Shangai Bank Corporation (HSBC), de capitales ingleses, y se autoriza nuevamente el funcionamiento del Banco Santander. Todavía no es posible evaluar la participación de estos bancos, pero suponemos que llegan con la intención de disputar una parte importante del mercado, donde solo tendrán preponderancia los bancos con mayor poder de mercado.

\section{LA PARTICIPACIÓN DE LA BANCA EXTRANJERA EN EL SISTEMA BAN- CARIO PERUANO}

\subsection{La propiedad de los bancos}

Los bancos extranjeros en el Perú han incrementado su presencia dentro de la estructura del sistema bancario, de forma relevante; de tal manera que observamos que, en 1998, participaban del 58\% del total de bancos; y en el 2007, constituyen el $77 \%$ del sistema. Mientras que, de otro lado, se tiene que la banca nacional pasa de una participación de 42\%, en 1998, a 23\%, en el 2007 (ver Cuadro 1). De estas proporciones, se colige la importancia que tiene en el sistema bancario este tipo de estructura de propiedad de la banca, basada ahora en la banca privada extranjera en todos sus niveles, es decir, a nivel de banca grande, mediana y pequeña.

Cuadro N. ${ }^{\circ}$ 1. Propiedad de los bancos en el Perú: 1998, 2001 y 2007.

\begin{tabular}{|l|c|c|c|c|c|c|}
\hline & $\mathbf{N}^{\circ}$ de bancos & $\mathbf{\%}$ & $\mathbf{N}^{\circ}$ de bancos & $\mathbf{\%}$ & $\mathbf{N}^{\circ}$ de bancos & $\mathbf{\%}$ \\
\hline Nacional & 11 & 42 & 4 & 36 & 3 & 23 \\
\hline Extranjera & 15 & 58 & 11 & 73 & 10 & 77 \\
\hline Total & 26 & 100 & 15 & 110 & 13 & 100 \\
\hline
\end{tabular}

Fuente: Superintendencia de Banca y Seguros.

Elaboración: Propia.

Para el presente avance del trabajo, se considera como bancos extranjeros a los Bancos Continental y Scotiabank (antes Banco Wiese y luego Wiese Sudameris), y como bancos nacionales a los Bancos de Crédito e Interbank. Asimismo, debemos señalar que al conjunto de estos bancos los clasificamos como bancos grandes para el presente análisis. 
Efectos de la presencia de la banca extranjera en la banca nacional del Perú

\subsection{Los Activos de la Banca Grande Extranjera y Nacional}

Los activos de la banca grande muestran que los cuatro bancos que conforman este grupo han incrementado la concentración en el mercado bancario peruano, desde 70.1\%, en 1995, a 86.3\%, en el 2007; pasando por el sub-periodo de 1996 a 1998, en donde dicha concentración disminuyó, debido al mayor número de bancos en el sistema (ver el Cuadro 2). Sin embargo, luego que salen del mercado seis bancos en el año 2000 y cinco bancos en el año 2001, se deja sentir el efecto de la menor competencia a través de la mayor concentración de los activos de este grupo de bancos.

El Banco de Crédito es el que mantiene la mayor proporción de activos totales, siguiendo el Banco Continental, Scotiabank e Interbank, en ese orden.

\section{Cuadro ${ }^{\circ} .^{\circ}$ 2. Banca extranjera y nacional en porcentajes.}

\begin{tabular}{|c|c|c|c|}
\hline \multirow{2}{*}{ Años } & \multicolumn{3}{|c|}{ Bancos Grandes } \\
\cline { 2 - 4 } & Extranjeros & Nacionales & Total \\
\hline 1995 & 34,8 & 35,3 & 70,1 \\
\hline 1996 & 33,4 & 35,9 & 69,3 \\
\hline 1997 & 32,0 & 33,0 & 65,0 \\
\hline 1998 & 30,2 & 32,9 & 63,1 \\
\hline 1999 & 35,7 & 32,0 & 67,7 \\
\hline 2000 & 39,9 & 33,3 & 73,2 \\
\hline 2001 & 40,0 & 34,6 & 74,6 \\
\hline 2002 & 36,8 & 38,5 & 75,3 \\
\hline 2003 & 39,1 & 42,1 & 81,2 \\
\hline 2004 & 39,5 & 41,6 & 81,1 \\
\hline 2005 & 40,1 & 43,7 & 83,8 \\
\hline 2006 & 40,4 & 45,5 & 85,9 \\
\hline 2007 & 40,1 & 46,2 & 86,3 \\
\hline Fuente: Superintendencia de Banca y Seguros. \\
\hline Elaboración: Propia. & & \\
\hline
\end{tabular}




\section{Gaby Cortez Cortez}

Los activos, tanto de la banca nacional como extranjera, disminuyen de 1996 a 1998, lo que es explicado principalmente por la crisis bancaria de esos años; y luego se observa que la banca extranjera incrementa sus activos de 1999 al 2000, manteniéndose, prácticamente, en este último nivel hasta el 2007. De otro lado, los activos de la banca nacional aumentan su participación dentro del total de activos del $2000 \mathrm{al} 2007$, superando a los activos de la banca extranjera (ver Gráfico 1).

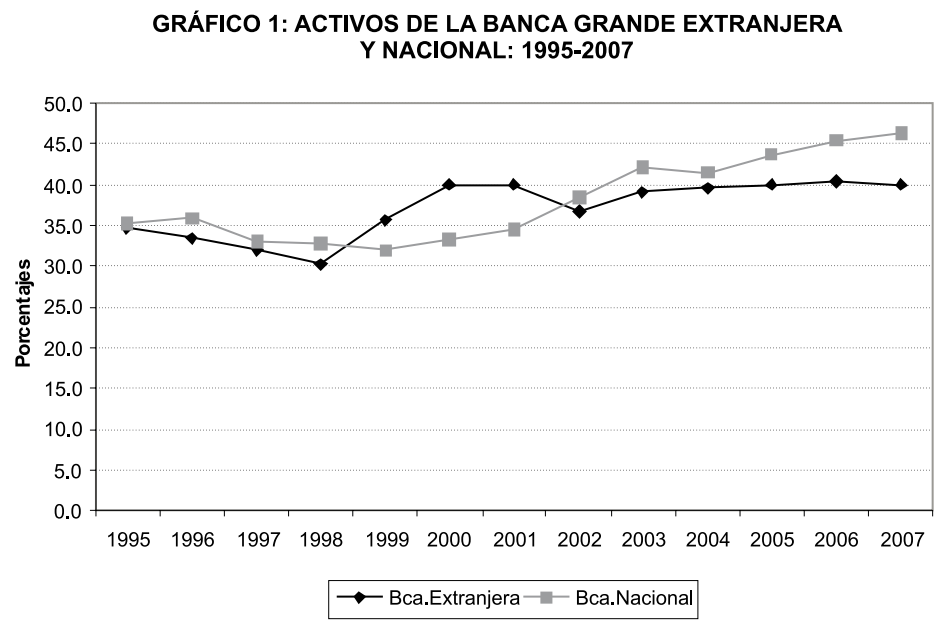

\subsection{El margen de los bancos grandes extranjeros y nacionales}

\subsubsection{Margen de los bancos extranjeros}

Un hecho importante que se encuentra es que el margen promedio de los bancos grandes ha disminuido de 1995 al 2007 de manera significativa (47\%), tal como puede observarse en el Cuadro 3. Contribuye de manera trascendente a la mengua del margen promedio la participación de la banca extranjera, principalmente a través del Banco Continental, ya que su margen es el más bajo de los bancos grandes inclusive, liderando de esta manera la reducción de este indicador en el sistema.

Debe indicarse que luego de la compra del Banco Continental del Perú por parte del Banco Bilbao Vizcaya Argentaria de España, le ha tomado varios años al Banco Continental BBVA posicionarse en la plaza peruana; $y$ lo hace paulatinamente a partir del año 


\section{efectos de la presencia de la banca extranjera en la banca nacional del Perú}

2001, mediante la disminución de su margen por debajo del margen promedio de los bancos grandes. La ventaja de este banco es que adquirió una marca local reconocida y bien posicionada en el medio a la que se le aplicó las ventajas de una banca corporativa con acceso al mercado financiero internacional.

De otro lado, se debe señalar que Scotiabank en los primeros años del análisis (Banco Wiese), mantenía márgenes más bajos que el resto de bancos hasta el año 2001, para luego incrementarse estos de manera importante del 2002 al 2004, probablemente como resultado de la venta de este banco al Banco Lima - Sudameris, cuya cartera combinada de activos y pasivos no ha debido ser fácil de administrar por los nuevos compradores extranjeros. Finalmente, el margen de este banco empieza a disminuir de cierta forma a partir del 2004, y luego con la compra del Banco Wiese-Sudameris por parte de Scotiabank, el margen se reduce más pronunciadamente, pero situándose todavía por encima del margen promedio de estos bancos, lo cual nos deja espacio para observar el funcionamiento de este nuevo banco extranjero grande en el sistema. La interrogante es: ¿Cuánto tiempo le tomará a Scotiabank mejorar su margen bancario?

Cuadro $\mathbf{N}^{\circ}{ }^{\circ}$ 3. Margen de la banca grande extranjera y nacional (1995-2007)

\begin{tabular}{|c|c|c|c|c|c|}
\hline Años & Crédito & Continental & Wiese-Scotia & Interbank & Promedio \\
\hline 1995 & 10,8 & 15,8 & 9,5 & 16,7 & 13,20 \\
\hline 1996 & 10,8 & 13,7 & 7,9 & 15,9 & 12,08 \\
\hline 1997 & 11,1 & 10,5 & 6,3 & 11,1 & 9,75 \\
\hline 1998 & 9,4 & 10,5 & 7,2 & 10,7 & 9,45 \\
\hline 1999 & 9,5 & 10,2 & 7,5 & 11,0 & 9,55 \\
\hline 2000 & 9,6 & 9,1 & 7,2 & 9,9 & 8,95 \\
\hline 2001 & 10,6 & 8,8 & 6,2 & 11,0 & 9,15 \\
\hline 2002 & 8,5 & 7,6 & 9,6 & 10,9 & 9,15 \\
\hline 2003 & 8,4 & 7,9 & 11,5 & 10,5 & 9,58 \\
\hline 2004 & 9,3 & 7,4 & 10,8 & 11,7 & 9,80 \\
\hline 2005 & 7,8 & 6,4 & 9,2 & 10,8 & 8,55 \\
\hline 2006 & 7,5 & 5,3 & 9 & 11,3 & 8,28 \\
\hline 2007 & 5,5 & 5,1 & 8,5 & 8,7 & 6,95 \\
\hline \multicolumn{7}{|l|}{ Fuente: Superintendencia de Banca y Seguros. / Elaboración: Propia. } \\
\end{tabular}




\section{Gaby Cortez Cortez}

y en una mayor eficiencia, lo que conlleva a su nueva posición de ventaja en el mercado bancario peruano (ver Gráfico 3).

De otro lado, Scotiabank, durante el periodo del Banco Wiese - Sudameris, muestra años con un ROE negativo y otros con niveles sumamente escasos. Estos resultados, unidos al efecto del bajo margen que ostentaban, nos permiten sugerir que tal institución estaba débil frente a la competencia de nuevos bancos, y trató de adecuarse para no perder espacio recortando su margen, pero que, lamentablemente, los problemas que enfrentó como banco nacional en ese momento no le permitieron continuar sus operaciones. Una prueba de ello es que luego del desgaste del Banco Wiese Sudameris, es adquirido por Scotiabank, e, inmediatamente, se mejoran los resultados en los años 2006 y 2007.

GRAFICO 3: ROE DE LOS BANCOS CONTINENTAL. Y DE CRÉDIT O: 1999-2007

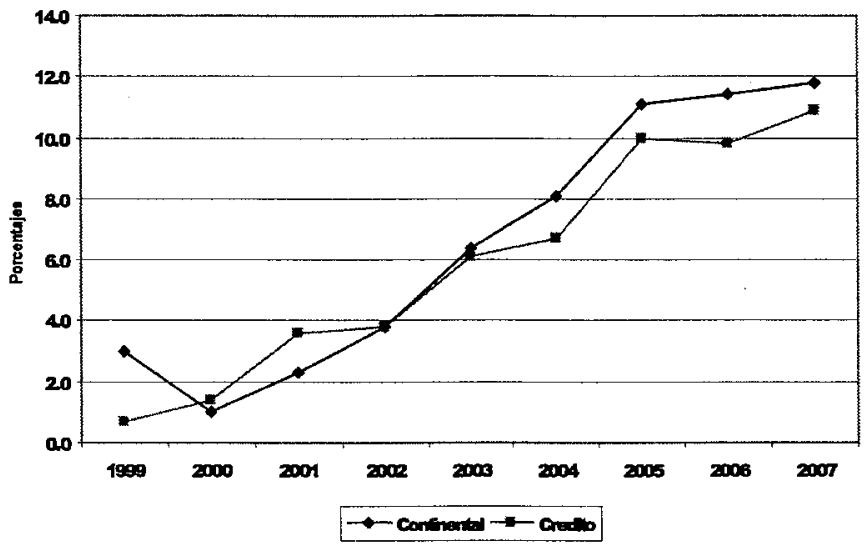

\section{CONCLUSIONES PRELIMINARES}

1. La estructura del sistema bancario peruano está constituida por el $77 \%$ de bancos extranjeros y el $23 \%$ de bancos nacionales en el año 2007 ; lo que significa que se ha pasado de una estructura de bancos estatales a una estructura de bancos extranjeros, fundamentalmente. 


\subsection{Rentabilidad de la banca grande extranjera y nacional}

La rentabilidad de los bancos grandes medida a través del ROE promedio ha tenido un comportamiento creciente, especialmente a partir del año 2002, y se acelera significativamente del 2005 al 2007, especialmente, tal como se puede apreciar en el Cuadro $\mathrm{N} .{ }^{\circ} 4$. Esto puede ser explicado, en gran parte, por la disminución de la competencia entre bancos, de manera que frente a un menor número de instituciones bancarias, se pudieron fijar mejores condiciones para los bancos, pero no para el público consumidor, lo que conduce por un hilo a mayores ganancias para los accionistas.

Cuadro N. ${ }^{\circ}$ 4. ROE de la Banca Grande Nacional y Extranjera: 1999-2007.

\begin{tabular}{|c|c|c|c|c|c|}
\hline Años & Continental & Crédito & Wiese-Scotia & Interbank & $\begin{array}{c}\text { Promedio } \\
\text { grupo }\end{array}$ \\
\hline 1999 & 3,0 & 0,7 & $-0,1$ & 0,4 & 1,0 \\
\hline 2000 & 1,0 & 1,4 & 0,5 & 0,3 & 0,8 \\
\hline 2001 & 2,3 & 3,6 & $-1,2$ & 1,1 & 1,5 \\
\hline 2002 & 3,8 & 3,8 & 0,8 & 2,4 & 2,7 \\
\hline 2003 & 6,4 & 6,1 & 0,4 & 5,2 & 4,5 \\
\hline 2004 & 8,1 & 6,7 & 0,5 & 4,7 & 5,0 \\
\hline 2005 & 11,1 & 10,0 & 1,6 & 7,9 & 7,7 \\
\hline 2006 & 11,4 & 9,8 & 6,1 & 9,6 & 9,2 \\
\hline 2007 & 11,8 & 10,9 & 9,9 & 11,3 & 11,0 \\
\hline $\begin{array}{c}\text { Promedio } \\
\text { por banco }\end{array}$ & 6,5 & 5,9 & 2,1 & 4,8 & 4,8 \\
\hline
\end{tabular}

Fuente: Superintendencia de Banca y Seguros.

Elaboración: Propia.

Se encuentra que durante estos años el Banco Continental ha tenido el promedio más alto de ROE, siguiéndole en segundo lugar el Banco de Crédito; en tercer lugar, Interbank; y, finalmente, en cuarto lugar, Scotiabank. Nuevamente, se observa que el Banco Continental lidera a los bancos grandes, en cuanto a rentabilidad del capital, adicionalmente a tener un margen bancario más bajo, a pesar de poseer un menor nivel de activos; mostrando de esta forma su buen manejo gerencial en la disminución de costos 


\section{Gaby Cortez Cortez}

\subsubsection{Margen de los bancos nacionales}

El Banco de Crédito es el mayor representante de los bancos nacionales grandes y ha mostrado un margen algo superior al promedio de este grupo de bancos de 1995 al 2001; mientras que del 2002 al 2007, nos señala una disminución de su margen respecto al promedio de estos bancos, situándose por debajo del margen promedio, lo que nos indica que tuvo que ajustar sus costos y mejorar su eficiencia frente a la presencia de otros bancos extranjeros.

También es importante destacar que el margen del Banco de Crédito, a pesar de haber disminuido en el fragor de la competencia, se ha situado por encima del nivel del Banco Continental, del 2000 al 2007, mostrándonos cierta debilidad en la competitividad de este banco nacional, e indicándonos una perdida de posición en el mercado.

De otro lado, el Banco Interbank es la institución que ha mostrado el margen bancario más alto durante el periodo de análisis, situándose este por encima del de todos los demás bancos y del promedio en su conjunto, revertiéndose dicha tendencia en el 2007 de manera pronunciada.

Es preciso hacer notar que el margen promedio de la banca nacional ha sido superior al de la banca extranjera de 1995 al 2007, indicándonos que ha sido la banca extranjera la que ha impulsado la disminución del margen bancario mediante la reducción de costos y una mayor eficiencia (ver Gráfico 2).

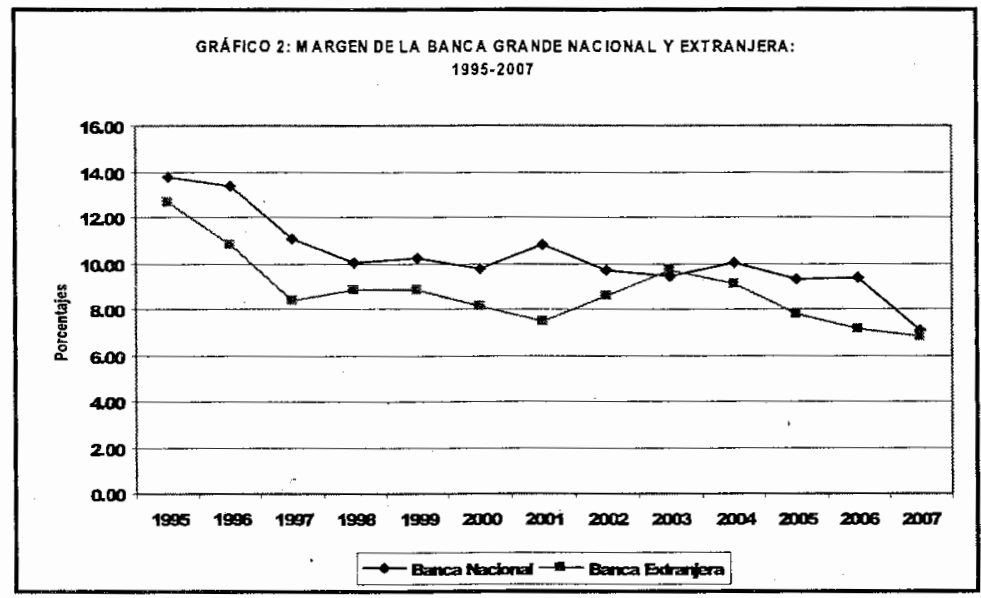




\section{Efectos de la presencia de la banca extranjera en la banca nacional del Perú}

2. Se observa que los activos de los bancos grandes nacionales -específicamente, a través del Banco de Crédito- controlan la mayor proporción de activos, lo que constituye su fortaleza, la que está basada en su conocimiento del mercado local.

3. La banca extranjera -específicamente a través del Banco Continental- lidera la disminución del margen bancario, en base a la disminución de costos y de mayor eficiencia en sus operaciones.

4. Frente a un menor número de bancos y a una disminución de la competencia, se observa un aumento significativo de las ganancias de los accionistas de los bancos, quienes ejercen su mayor poder de mercado.

\section{BIBLIOGRAFÍA}

Lensink, R., Hermes, N. (2002)."The short - term effects of foreign bank entry on domestic bank behavior: Does economic development matter?". Journal of Banking \& Finance. 2002.

Okuda, H., Rungsomboon, S. (2006). "Comparative cost study of foreign and Thai domestic banks in 1990-2002: Its policy implications for a desirable bankng industry structure". Journal of Asian Studies.

Pasiouras, F., Kosmidou, K. (2007). Factors influencing the profitability of domestic and foreign comercial banks in the European Union. Research in Internacional Business and Finance.

Van Tassel, E., Vishwasrao, S. (2007). "Asymmetric information and the mode of entry in foreign credit markets". Journal of Banking \& Finance.

Unite, A, Sullivan, M. (2003). The effect of foreign entry and ownership structure on the Phillipine domestic banking market. Journal of Banking \& Finance.

Yeyati, E., Micco, A. (2007). "Concentration and foreign penetration in Latin American banking sectors: Impact on competition and risk". Journal of Banking \& Finance. 